\title{
A Synergistic Approach of Injectable-Platelet Rich Fibrin and Bio Stimulation in the Treatment of Oral Lichen Planus
}

Dr. Archana Shankar, MDS ${ }^{1}$, Dr. Sheela Kumar Gujjari, MDS ${ }^{2 *}$, Dr. Jaishankar HP MDS ${ }^{3}$, Dr. Minusha Grace $\mathrm{S}^{4}$

${ }^{1}$ JSS, Dental College and Hospital, Mysuru, Karnataka, India

${ }^{2}$ HOD, Department of Periodontology, JSS Dental College and Hospital, Mysuru, Karnataka, India

${ }^{3}$ Reader, Department of Oral medicine And Radiology, JSS dental College and Hospital, Mysuru, Karnataka, India

${ }^{4}$ MDS-Second Year student JSS dental College and Hospital , Mysuru, Karnataka, India

DOI: $\underline{\text { 10.36348/sjodr.2021.v06i02.006 }}$ | Received: 11.02 .2021 | Accepted: 22.02 .2021 | Published: 26.02 .2021

\section{Abstract}

Oral Lichen planus is a mucocutaneous disease of autoimmune origin having a prevalence of 0.02 percent in India and affecting women more [1]. It is a perplexing condition and poses dilemma to the dentist in providing the apt treatment to the patient. Though various treatment modalities are available steroids is the most commonly prescribed, Bio stimulation (Low Level Laser therapy) has been found to provide pain relief. Recently Platelet concentrates have been researched of which injectable form (i-PRF) has been found to be promising. The beneficial effects of bio stimulation and i-PRF have not yet been studied. So, an effort was made to combine both to achieve a successful, non-invasive technique has been reported in this case report. There was improvement in Oral manifestation and Quality of life of the Patient

Keywords: Oral Lichen planus, Low Level Laser Therapy, i-PRF, Visual Analog Scale.

Copyright (C) 2021 The Author(s): This is an open-access article distributed under the terms of the Creative Commons Attribution 4.0 International License (CC BY-NC 4.0) which permits unrestricted use, distribution, and reproduction in any medium for non-commercial use provided the original author and source are credited.

\section{INTRODUCTION}

The etiology of Lichen planus is multifactorial and presents in various forms such as reticular, papular, erosive, plaque like and bullous forms [2]. They are commonly seen in the buccal mucosa, Gingiva and Dorsum of the tongue [3]. The clinical diagnosis can be confirmed using an oral biopsy and use of direct immunofluorescence [3]. It's a chronic premalignant condition which causes pain discomfort and inability to maintain oral hygiene. A plethora of treatment modalities have been in practice ranging from use of immunosuppressive drugs $h$ Laser therapy. Though corticosteroids appear to be the most effective treatment, over a long duration it can cause candidiasis, xerostomia, delayed wound healing, hyperglycaemia, adrenal insufficiency etc [4]. So, options like bio stimulation using Low Level Laser therapy have been found to reduce the size of lesion with complete remission of burning sensation. Of late there has been an interest in using platelet concentrates such as PRF due to the release of growth Factors. There is a new form of PRF, that is injectable PRF (i-PRF) of which there are hardly any studies. But it has the advantage of enhanced wound healing, improves angiogenesis and its mode of application is more specific. So, expecting a synergistic effect of bio stimulation and i-PRF this approach was chosen as the treatment modality for the Patient.

\section{CASE REPORT}

A 40-year-old male patient had reported to the dental OPD with the complaint of pain and burning sensation of the gums and buccal mucosa since 1 month. The pain aggravated on consumption of hot and spicy food. Patient claimed to be systemically healthy and not on any medications. He was a non-smoker and non-alcoholic. Intra oral examination revealed poor oral hygiene and Wickham's striae was seen on the left and right buccal mucosa, extending with relation to 24 to 27 region and 34 to 37 region and in 44 to 47 gingival regions.(Fig1) The lesion was evaluated using Thongprasom sign scoring system [6] and was found to be score 4 (Table-1). Based on the clinical examination, case was diagnosed as reticular lichen planus. The haematological investigation was found to be normal. Visual Analog Scale (VAS) using Wong-Baker faces pain rating score [7] was recorded (Table-2). Scaling and Root planing was performed and Oral Hygiene instructions given. Patient was advised Topinate gel, Clobetasol for topical application for 15 days and capsule Lycored twice daily for 15 days. Patient was recalled after 15 days. VAS score and clinical 
examination revealed no improvement so it was decided to use i-PRF with bio stimulation (Fig2). The procedure was explained to the patient and consent taken. $10 \mathrm{ml}$ of venous blood was withdrawn and centrifuged at 700 rpm for 3 minutes. The resultant i-PRF was taken in insulin syringes and injected into the affected regions. Patient was treated using Sunny Diode Laser (Fig3) which is a semiconductor diode with laser source that emits invisible infrared laser light at $808 \mathrm{~nm}$ wavelength. It was used in a non-contact mode using fibre optic fibre of diameter $600 \mu \mathrm{m}$ at $1 \mathrm{~J}, 0.8 \mathrm{~W}$ for $15-$ 20 seconds in 3cyclestsessions in the affected areas. This procedure was repeated thrice in 15 days gap. The patient was recalled after 3 months and it was observed that there was improvement in the second sitting. After 6 months when the patient was reviewed it was observed there was resolution of the lesion as seen by the Throngorasom score which reduced from 4 to 1 and also the VAS Scale showed reduction in pain from 8 to 2.

\section{DISCUSSION}

Lichen planus is multifactorial and is a chronic condition with remission and exacerbation. Though topical steroids such as Clobetasol 17- Propionate have shown improvement in some patients, there was no relief found after 15 days of application. This could have been due to the saliva volume and the tongue movements which could have decreased the effectiveness of the therapy. Long term use of Clobetasol can lead to tachyphylaxis and adrenal insufficiency [8]. So, treatment modalities with less side effect such as LASER and i-PRF was opted. i-PRF showed good result in this patient as there was resolution in the lesion. This could have been due to angiogenesis, immunomodulation and the capacity of iPRF to release growth factors which helps in wound healing. Very sparse literature is their regarding the use of i-PRF in the treatment of oral lichen Planus so it needs to be researched. The results of this case report was similar to the results obtained in a study which compared i-PRF and Corticosteroids [9]. In this study there was improvement in the VAS score, Thongprasom scoring and the Quality of Life, as seen in other studies where Low level Laser therapy used [11-13] and The reduction in pain after Laser providing analgesic effect by improving endorphin release and inhibiting nociceptive signals as well as by controlling Pain mediators.

\section{CONCLUSION}

The Combination of LLLT and i-PRF have shown a drastic improvement in this Patient and changed his Quality of Life as seen by the VAS scale, Thongprasom score. The results of these synergistic treatment modalities, LLLT and i-PRF have been promising but more studies are required to prove its potential.

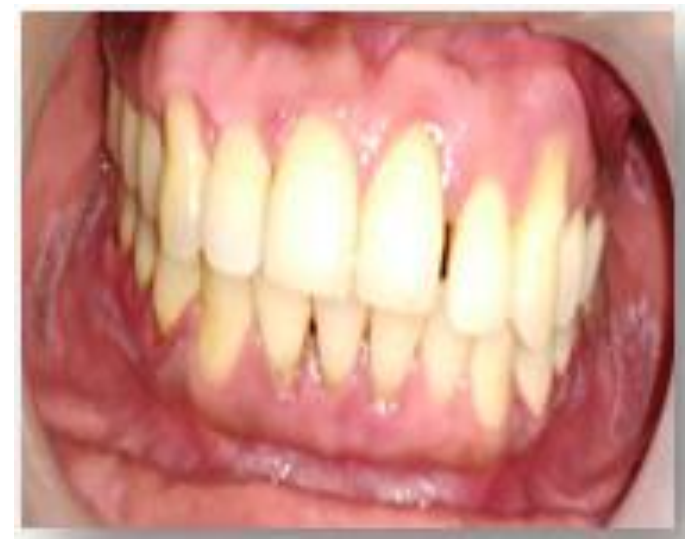

Fig-1: Preoperative

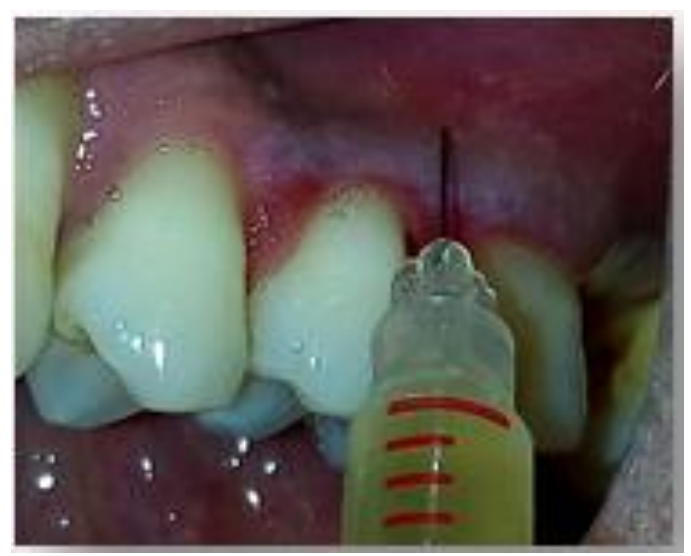

Fig-2: Injecting i-prf into affected sites

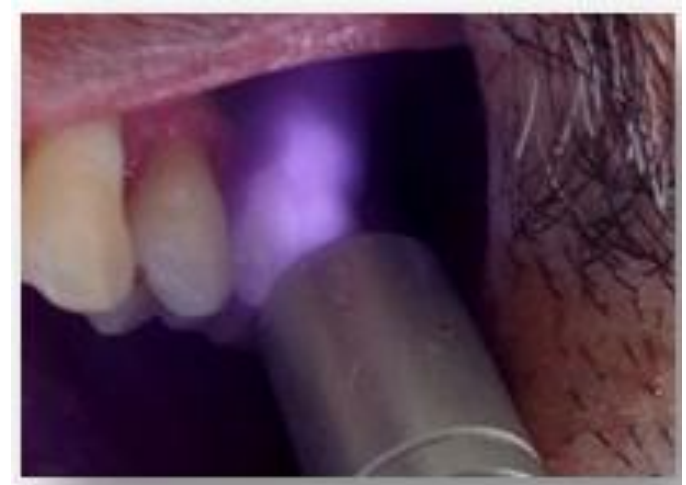

Fig-3: Laser biostimulation at 600nm using diode

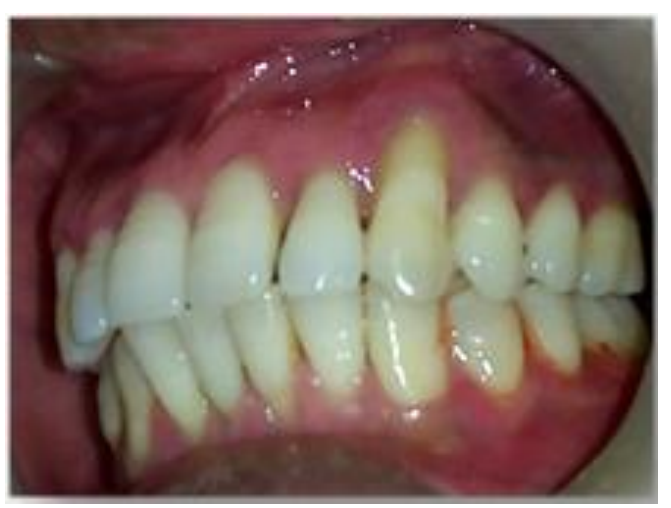

Fig-1: Postoperative 
Table-1

\begin{tabular}{|c|c|}
\hline Score & Characteristics \\
\hline 5 & $\begin{array}{l}\text { white striae with erosive area } \geq 1 \\
\qquad \mathrm{~cm} 2\end{array}$ \\
\hline 4 & $\begin{array}{l}\text { white striae with erosive area }<1 \\
\qquad \mathrm{~cm} 2\end{array}$ \\
\hline 3 & $\begin{array}{l}\text { white striae with atrophic area } \geq 1 \\
\qquad \mathrm{~cm} 2\end{array}$ \\
\hline 2 & $\begin{array}{l}\text { white striae with atrophic area }<1 \\
\qquad \mathrm{~cm} 2\end{array}$ \\
\hline 1 & mild white striae only \\
\hline
\end{tabular}

Table-2

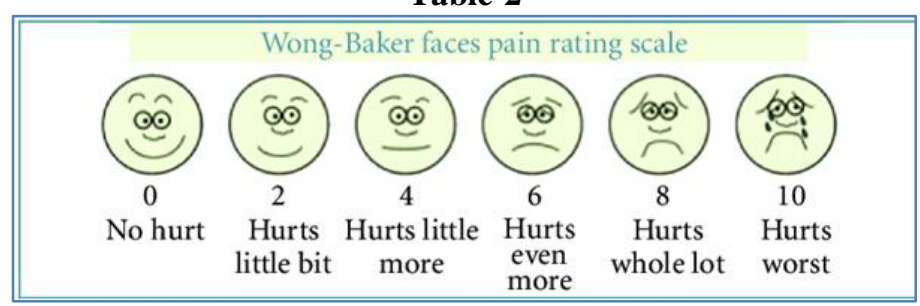

\section{REFERENCES}

1. MDedge.com-internet references

2. Lavanya, N., Jayanthi, P., Rao, U. K., \& Ranganathan, K. (2011). Oral lichen planus: An update on pathogenesis and treatment. Journal of oral and maxillofacial pathology: JOMFP, 15(2), 127.

3. Srinivas, K., Aravinda, K., Ratnakar, P., Nigam, N., \& Gupta, S. (2011). Oral lichen planus-Review on etiopathogenesis. National journal of maxillofacial surgery, 2(1), 15.

4. Karagoz, G., Bektas-Kayhan, K., \& Unur, M. (2016). Desquamative gingivitis: A review. Journal of Istanbul University Faculty of Dentistry, 50(2), 54.

5. Mahdavi, O., Boostani, N., Jajarm, H. H., Falaki, F., \& Tabesh, A. (2013). Use of low level laser therapy for oral lichen planus: report of two cases. Journal of Dentistry, 14(4), 201.

6. Miron, R. J., Fujioka-Kobayashi, M., Hernandez, M., Kandalam, U., Zhang, Y., Ghanaati, S., \& Choukroun, J. (2017). Injectable platelet rich fibrin (i-PRF): opportunities in regenerative dentistry?. Clinical oral investigations, 21(8), 2619-2627.

7. Pakfetrat, A., Delavarian, Z., Falaki, F., Khorashadizadeh, M., \& Saba, M. (2015). The effect of pimecrolimus cream $1 \%$ compared with triamcinolone acetonide paste in treatment of atrophic-erosive oral lichen planus. Iranian journal of otorhinolaryngology, 27(79), 119.
8. Bijina Rajan, J. A., Shenoy, N., Denny, C., Ongole, R., \& Binnal, A. (2014). Assessment of quality of life in patients with chronic oral mucosal diseases: a questionnaire-based study. The Permanente Journal, 18(1), e123.

9. Khatri, A., \& Kalra, N. (2012). A comparison of two pain scales in the assessment of dental pain in East Delhi children. International Scholarly Research Notices, 2012.

10. Tunali, M. U. S. T. A. F. A., Ozsagir, Z. B., Unver, T., Saglam, E., Alinca, S. B., \& Toprak, A. (2018). A new treatment approach for patients with oral lichen planus; I-PRF. J Clin Periodontol, 45, 84-5.

11. Elshenawy, H.M., Eldin, A.M., Abdelmonem, M.A. (2015). Clinical Assessment of the Efficiency of Low-Level Laser Therapy in the Treatment of Oral Lichen Planus. Open Access Maced J Med Sci, 3(4):717-21.

12. Patil, A., Prasad, S., Ashok, L., \& Sujatha, G. P. (2012). Oral bullous lichen planus: Case report and review of management. Contemporary clinical dentistry, 3(3), 344.

13. Suman, S.V., Nagajyothi Peta, Vijaykumar Bokkasam, Sathish Babu. D, Sunil. Y.V., Yamini, P. (2019). Management of pain in erosive lichen planus using low level laser therapy (LLLT): a report of two cases. International Journal of Contemporary Medicine Surgery and Radiology, 4(3)

14. Adriana, C., Gianni, A., Paolo, G. (2010). Arduino, Carbone Mario, Gianluca Massolini, Marco Mozzati, and Roberto Broccoletti. Photomedicine and Laser Surgery, S-99-S-103. 